\title{
Study on the determination of sodium cyclamate in the jelly
}

\author{
Ren Boru \\ College of Supervision of Quality\&Technology, Hebei \\ University;Xingtai Qiaoxi Food and Drug Administration \\ China,Baoding,Xingtai \\ Liu Na \\ Logistics Services Group Catering Service Center,Heibei \\ Normal University \\ China, Shijiazhuang
}

*Corresponding author : Wang Tingxin

\author{
Zhao Jie \\ College of Supervision of Quality\&Technology, Hebei \\ University \\ China,Baoding \\ Wang Tingxin* \\ College of Supervision of Quality\&Technology, Hebei \\ University \\ China,Baoding
}

\begin{abstract}
Objective: In order to study the contect of sodium cyclamate in jellies. Method: Using ring amino sulfonate sodium hypochlorite derived reaction occurs under acidic conditions to generate $\mathrm{N}$-, $\mathrm{N}$-dichloro-cyclohexane, and then hexane extracted ODC-C14 column,methanol $(\mathrm{V})$ :water $(\mathrm{V})=\mathbf{8 0 : 2 0}$ as mobile phase separation,the flow rate was $1.0 \mathrm{~mL} / \mathrm{min}$, the injection volume was $20 \mathrm{ul}$,the detection wavelength of $314 \mathrm{~nm}$. Result This high-efficiency liquid chromatography determination of the concentration of sodium cyclamate in the linear relationship between the $0-300 \mathrm{ug} / \mathrm{ml}$,the correlation coefficient was 0.9995 above, and require accuracy and good reproducibility. The recovery rate of sodium cyclamate was $\mathbf{8 0 \% - 1 2 0 \%}$, the coefficient of variation less than 10\%.Conclusion:The method is rapid, simple,highly sensitive and with less interference, which is suitable for the detection of cyclamate in jellies.
\end{abstract}

Keywords -sodium cyclamate;jelly; high efficiency liquid chromatography;detection

\section{INTRODUCTION}

Sodium cyclamate is a non nutritive sweeteners, and it is commonly used in pickles class,sauces,cakes,wine ,beverages and other foods [1-2] .Sodium cyclamate was forbidden to add in food because it has carcinogenic,teratogenic,impaired renal function and so on such side effects [3-4] . It is harmful to our health for the long-term excessive consumption of sodium cyclamate [5] .

At present, many researches has been focused on the determination of sodium cyclamate. The volatile chemical properties of cyclohexanol nitrous acid ester is used to determine the content of sodium cyclamate in yogurt, ice cream, candied dates, with headspace gas chromatography method, and the content of sodium cyclamate and benzoic acid in food can also be determined simultaneously [6-9] . Qian Guiping et al. determined the content of sodium cyclamate using tro-electrode system. The secondary derivative polarographic wave is measured in ammonia solution at $-0.38 \mathrm{~V}$. The peak potential is used to determine the sodium cyclamate qualitatively, and the peak height is used to determine the sodium cyclamate quantitatively [10] .In this paper, a high-efficiency liquid chromatography method to determine the content of cyclamate in jelly is proposed based on these reported studies, which is applicable in the quality assessment system of jelly.

\section{II.MATERIALS AND METHODS:}

\section{A. Instrument:}

1) High performance liquid chromatography: 883 Basic IC plus, The Switzerland Metrohm Company, equipped with a conductivity detector/chromatographic module and chromatography workstation,suppressor,tandem pump;

2) Ultra pure water instrument:NEX UP type 1000,South Korea HUMAN Company;

3) High speed centrifuge: China YaRong Co. ltd.;

4) Numerical control ultrasonic cleaning instrument:type of KQ-500 DE, KunShan Ultrasonic Instrument Co. ltd.;

5) Electronic balance:type of AR1140,METTLER-TOLEDO Instruments (Shanghai) Co. ltd.;

6) Water bath: type of SY1-P3S, Beijing Oriental JingRui Technology Development Co., ltd..

\section{B. The sample and reagent:}

Sample: jelly, provided by the Guangdong XiZhiLang Co. ltd.;

The reagent: methanol (Chromatographic pure); N-hexane; sodium hypochlorite; sodium bicarbonate;concentrated sulfuric acid(98\%); all reagents were provided by Baoding BoaAiXin reagent company. 


\section{Chromatographic conditions:}

Conlumn:ODS-C18(250*4.6mm);

Suppressor:Automatic generating suppressor,with the removal of carbon dioxide function;

Sample size:20uL;

Wavelength:314nm.

\section{The preparation of standard solution:}

The preparation of the original solution: Weigh $0.1000 \mathrm{~g}$ sodium cyclamate accurately in volumetric flask of $50 \mathrm{ml}$ constant volume, the concentration of original solution is $2 \mathrm{mg} / \mathrm{ml}$.

The preparation of standard solution: remove $30 \mathrm{uL}, 65 \mathrm{uL}, 125 \mathrm{uL}, 250 \mathrm{uL}, 1000 \mathrm{uL}$ respectively in volumetric flask of $10 \mathrm{~mL}$ constant volume, the concentration of standard solution is 6mg/L,13mg/L,25mg/L,50mg/L,200mg/L.

\section{E. Standard solution:}

Measure $5 \mathrm{~mL}$ standard solution accurately in a separatory funnel,add $1 \mathrm{~mL}$ sulfuric acid(1:1),2.5mL $\mathrm{N}$-hexane, $0.5 \mathrm{~mL}$ sodium hypochlorite(1:1), shake for 1 minute,layer and abandon the lower,add $5 \% \quad 12.5 \mathrm{~mL}$ sodium bicarbonate in $\mathrm{N}$-hexane,shake for 1 minute,discard the lower, the experiment need upper $\mathrm{N}$-hexane layer .

\section{F. Sample pretreatment:}

Weigh $40.1245 \mathrm{~g}$ jelly samples accurately in a beaker,add $50 \mathrm{~mL}$ water, put into the numerical control ultrasonic cleaning instrument ultrasound 30 minutes, and then place in boiling water bath heat 5 minutes, after cool volumed in volumetric flask of $200 \mathrm{~mL}$. $40 \mathrm{~mL}$ solution centrifuge at $3500 \mathrm{rpm}$ for 5 minutes, take $20 \mathrm{~mL}$ supernatant,volume in volumetric flask of $50 \mathrm{~mL}$. The solution is processed according to the standard solution treatment method, and then inject into instruments.
Detector: conductivity detector, current $50 \mathrm{~mA}$. Detector temperature, room temperature;

Eluent: methanol(V): water $(\mathrm{V})=80$ : 20 , the flow rate was $1.0 \mathrm{~mL} / \mathrm{min}$;

\section{G. Determination of recovery rate and precision:}

Select two jelly samples,one with a standard solution(the final concentration of solition is $18 \mathrm{mg} / \mathrm{L}$ ), another as contrast. Measure the concentration of sodium cyclamate in high-efficiency liquid chromatograph according to the method of treatment E Calculate the recovery rate and coefficient of variation through formula. The recovery rate $=$ (the concentration of adding standard material samples-sample concentration) $/ 18 * 100$; coefficient of variation $\mathrm{RSD} \%=$ standard deviation/the concentration of sample mean.

\section{III.RESULT:}

\section{A. Standard curve of sodium cyclamate:}

Prepare a series of standard solutions according to standard method,then analyse under the above chromatographic conditions, do regression curve with concentration on the peak area.The experimental data shown in table 1 .The standard curve of sodium cyclamate shown in figure 2.

\section{$B$. The recovery of sodium cyclamate:}

Select jelly sample as the blank sample,add a certain concentration of sodium cyclamate standard solution (the final concentration of $18 \mathrm{mg} / \mathrm{L}$ ), mix fully,determine according to this experiment method,six groups of parallel.The measured sodium cyclamate recovery rate shown in table 3 .

TABLE I. THE EXPERIMENTAL DATA OF CYCLAMATE STANDARD SOLUTION

\begin{tabular}{|c|c|c|c|}
\hline $\begin{array}{c}\text { The concentration of } \\
\text { sodium cyclamate } \\
(\mathrm{mg} / \mathrm{L})\end{array}$ & The measure of area 1 & The measure of area 2 & The average of area \\
\hline 6 & 28378 & 31629 & 30003.5 \\
\hline 13 & 53089 & 51124 & 52106.5 \\
\hline 25 & 98393 & 109278 & 103835.5 \\
\hline 50 & 173220 & 206638 & 189929 \\
\hline 200 & 852385 & 860239 & 856312 \\
\hline
\end{tabular}




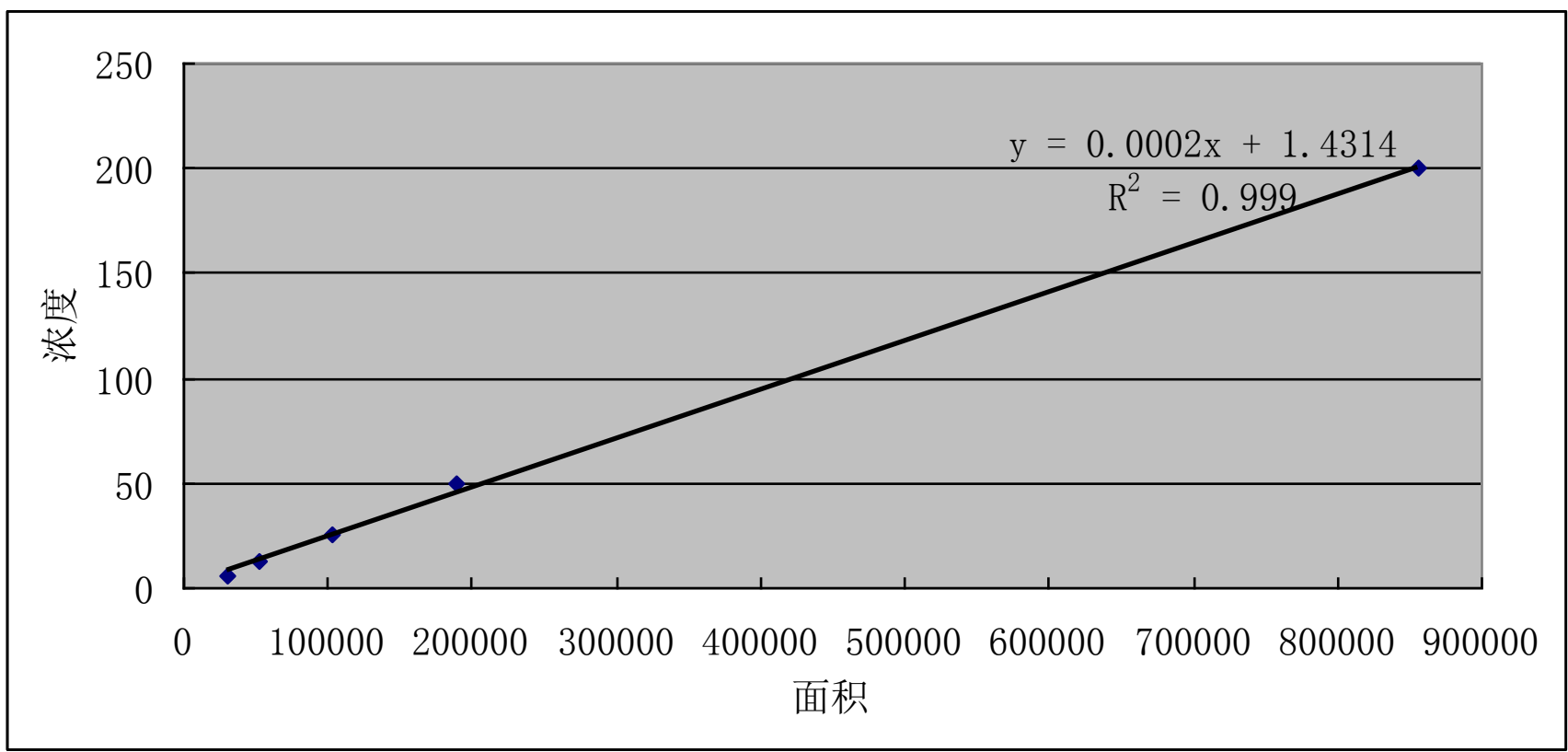

Figure 1.Standard curve of sodium cyclamate

TABLE II.THE RECOVERY OF SODIUM CYCLAMATE MEASURING BY LIQUID CHROMATOGRAPH

\begin{tabular}{|c|c|c|c|c|c|}
\hline material & $\begin{array}{l}\text { the samples } \\
(\mathrm{mg} / \mathrm{L})\end{array}$ & $\begin{array}{c}\text { standard } \\
\text { materia }(\mathrm{mg} / \mathrm{L})\end{array}$ & $\begin{array}{c}\text { Measured } \\
\text { amount } \\
(\mathrm{mg} / \mathrm{L})\end{array}$ & $\begin{array}{r}\text { Rate of } \\
\text { recovery }(\%)\end{array}$ & $\begin{array}{c}\text { The mean } \\
\text { recovery rate }(\%)\end{array}$ \\
\hline \multirow{6}{*}{$\begin{array}{l}\text { Sodium } \\
\text { cyclamate }\end{array}$} & 18.91 & \multirow{6}{*}{18} & 34.22 & 0.86 & \multirow{6}{*}{84.5} \\
\hline & 18.82 & & 33.80 & 0.83 & \\
\hline & 19.49 & & 33.76 & 0.83 & \\
\hline & 18.94 & & 34.53 & 0.87 & \\
\hline & 18.52 & & 33.93 & 0.84 & \\
\hline & 18.20 & & 33.85 & 0.84 & \\
\hline
\end{tabular}

The recovery rate of sodium cyclamate between $80 \%-120 \%$,illustrate the earlier treatment and determination method is accurate and feasible, which can carry on the accurate quantitative analysis.

C. Cyclamate variability(RSD\%) 
CYCLAMATE VARIABILITY(RSD\%)

\begin{tabular}{|c|c|c|c|c|c|c|c|}
\hline material & 1 & 2 & 3 & 4 & 5 & 6 & RSD\% \\
\hline $\begin{array}{l}\text { Sodium } \\
\text { cyclamate }\end{array}$ & 18.91 & 18.82 & 19.49 & 18.94 & 8.52 & 18.20 & 2.3 \\
\hline
\end{tabular}

We need to analyse the experimental data, High Efficiency liquid chromatography measuring Cyclamate variability shown in table 4 . The cyclamate of jelly samples in RSD\% is $2.3 \%$,the variability less than $10 \%$. Illustrate the method precision is good,can meet the requirements of analysis.

\section{IV.CONCLUSION:}

The method presented in this paper is rapid, simple,highly sensitive and with less interference, which is suitable for the detection of the quality of jelly for the national quality supervision department and can also provide timely reliability criterion for the quality control of jelly and other food. This method is expected to bring certain economic benefits and good social benefits and may provide better security guarantees for consumers.

\section{REFERENCES}

[1] GB/T 5009.97-2003.Determination of solium cyclamate in food.The national standard of the people'sRepublic of China.
[3] Bopp B A, Sonders R C , Kesterson J W. T oxicological aspects of cyclamate and cyclohexylamine[J ] . Crit Rev Toxicol ,1986 , 16 (3) :213

$-306$.

[4]Wangqi.Preliminary study on the development trend of jelly products and related technical problems[J].Science and technology of food industry,2001(4):77-78.

[5]Hanhua,Jiyuanbing,Lijili.Advances in research on the method for determination of solium cyclamate in food[J].Green science and technology,2013,(3):148-150.

[6]Houxiaoyan,Liyongfang,Lijunling. The determination of cyclamate in food with colorimetric tube headspace gas chromatographic method[J].China Medical Journal, 2004,14(4):497-498.

[7]Zhongzhixiong,Liangchunsui,Yaojing.The determination of sodium cyclamate and benzoic acid in food with lon chromatography method[J]. China Medical Journal,2005,15(9):1062-1064.

[8] Z, Y Y. A rapid separation and quantitation of sodium cyclamate in food reversed phase high efficiency liquid chromatography $\mathrm{Li}$ in $[\mathrm{J}]$. Se Pu , 1999 ,17 (3):278 - 279.

[9]Guoyingying,Zhuyan,Yeliming.Determination of sweeteners with eluent generator ion chromatography suppressed conductivity detection[J].Journal of Zhejiang University(natural science edition).2004,31(4):435-437.

[10]Qianguiping,Yujianzhong.Single Sweep Polarographic method for the determination of sodium cyclamate in food[J]. China Medical Journal,2000,10(6):692-694. 